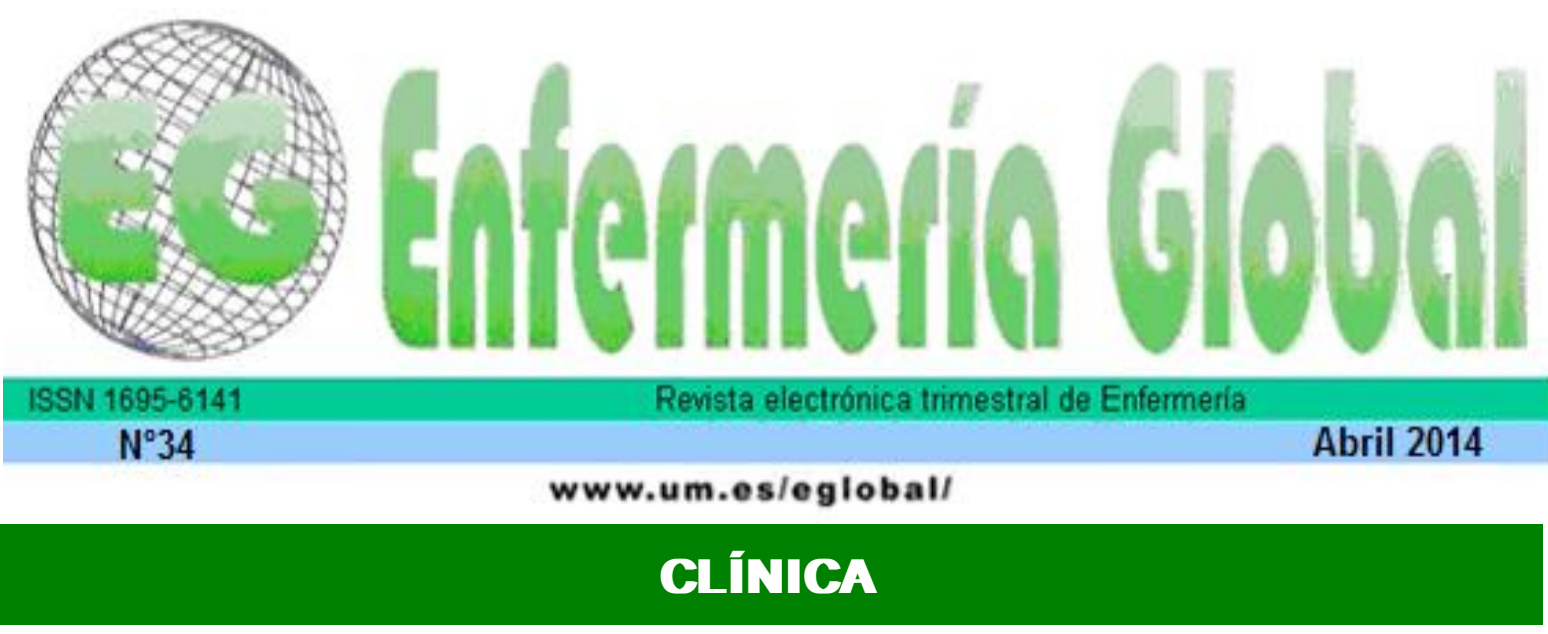

\title{
Diagnósticos de Enfermería en Pacientes Sometidos a Hemodiálisis
}

Diagnósticos de enfermagem em pacientes submetidos à hemodiálise

Nursing Diagnoses in Patients Undergoing Hemodialysis

\section{${ }^{*}$ Poveda, Vanessa de Brito ${ }^{* *}$ Alves, Juliana da Silva ${ }^{* *}$ Santos, Elaine de Freitas *** Moreira, Alessandra Garcia Emerick}

\author{
*Pos-Doctorado en Enfermería. Profesora de las Facultades Integradas Teresa D' Ávila (FATEA) y \\ Universidad de Vale do Paraíba (UNIVAP). E-mail: vbpoveda@gmail.com ${ }^{* *}$ Enfermera. \\ ${ }^{* * *}$ Enfermera. Profesora de la UNIVAP. Brasil.
}

Palabras clave: enfermería; diálisis renal; diagnóstico de enfermería

Palavras chave: enfermagem; diálise renal; diagnóstico de enfermagem

Keywords: nursing; renal dialysis; nursing diagnoses.

\section{RESUMEN}

Objetivo: Determinar los más comunes diagnósticos de enfermería en pacientes sometidos a tratamiento de hemodiálisis, basados en la nomenclatura de la North American Nursing Diagnosis Association - International (NANDA-I) 2009-2011.

Método: Este es un estudio cuantitativo, de tipo descriptivo exploratorio, realizado en una clínica de hemodiálisis en el estado de São Paulo, Brasil, con una muestra de 50 pacientes. El estudio fue aprobado por el Comité de Ética de la Investigación de la Universidad del Vale do Paraíba, bajo el protocolo $\mathrm{N}^{\circ} \mathrm{H} 236 / \mathrm{CEP} / 2009$.

Resultados: Se identificaron 24 diagnósticos más frecuentes, seis de los cuales se encontraron en $100 \%$ de la muestra estudiada; estos eran: eliminación urinaria afectada; integridad de la piel afectada; riesgo de infección; riesgo de perfusión renal ineficaz; movilidad física reducida; y riesgo de desequilibrio electrolítico.

Conclusión: La determinación de los diagnósticos de enfermería comunes en los sujetos sometidos a hemodiálisis ayudará a los profesionales de enfermería en la atención a los pacientes renales crónicos proporcionando herramientas para la planificación de la asistencia. 


\section{RESUMO}

Objetivo: determinar os diagnósticos de enfermagem mais frequentes em pacientes submetidos a tratamento hemodialítico, baseados na nomenclatura da North American Nursing Diagnosis Association-Internacional (NANDA-I) 2009-2011.

Método: trata-se de estudo quantitativo, do tipo descritivo exploratório, realizado em uma clínica de hemodiálise no interior do estado de São Paulo, com uma amostra de 50 pacientes. O estudo foi aprovado pelo Comitê de Ética em Pesquisa da Universidade do Vale do Paraíba sob número $\mathrm{H} 236 / \mathrm{CEP} / 2009$.

Resultados: foram identificados 24 diagnósticos mais frequentes, sendo seis encontrados em $100 \%$ da amostra estudada: eliminação urinária prejudicada, integridade da pele prejudicada, risco de infecção, risco de perfusão renal ineficaz, mobilidade física prejudicada e risco de desequilíbrio eletrolítico.

Conclusão: o estabelecimento dos diagnósticos de enfermagem comuns aos sujeitos submetidos a hemodiálise auxiliará o profissional de enfermagem envolvido no atendimento aos pacientes renais crônicos, fornecendo ferramentas para o planejamento da assistência.

\section{ABSTRACT}

Objective: To determine the most common nursing diagnoses in patients under hemodialysis treatment, based on the nomenclature of the North American Nursing Diagnosis Association International (NANDA-I) 2009-2011.

Method: This is a quantitative, descriptive and exploratory study, accomplished in a hemodialysis clinic in the State of São Paulo, Brazil, with a sample of 50 patients. The study was approved by the Committee of Ethics in Research of the University of Vale do Paraíba, under protocol No. $\mathrm{H} 236 / \mathrm{CEP} / 2009$.

Results: We identified 24 most frequent diagnoses, of which six were found in $100 \%$ of the sample studied; they were: impaired urinary elimination; impaired skin integrity; risk of infection; risk of ineffective renal perfusion; impaired physical mobility; and risk of electrolyte imbalance.

Conclusion: Determining nursing diagnoses common to subjects submitted to hemodialysis will help nursing professionals deal with chronic renal patients care by providing tools for planning assistance.

\section{INTRODUCCIÓN}

La Insuficiencia Renal Crónica (IRC) se caracteriza por la pérdida progresiva e irreversible de la función renal, teniedo como causas principales la diabetes mellitus y la hipertensión arterial $^{(1)}$.

La hemodiálisis consiste en un proceso de filtraje y depuración de la sangre de substancias no soportables como la creatinina y la urea, que han de ser eliminadas por el organismo deficiente en la realización de esta función ${ }^{(2)}$. El procedimiento se realiza de dos a cuatro veces a la semana, con duración de entre dos y cuatro horas.

La dedicación al tratamiento de la IRC por medio de la hemodiálisis acarrea limitaciones que interfieren en la rutina diaria de los pacientes, como la pérdida de empleo, alteraciones en la imagen corporal, restricciones dietéticas e hídricas ${ }^{(3)}$.

Así, tanto el enfermero como el responsable del equipo de enfermería, tienen un papel fundamental, al coordinar la asistencia prestada, persiguiendo una mejor 
calidad de vida de estos pacientes, enfatizando en un cuidado personalizado en este proceso $^{(4)}$.

El equipo de enfermería durante la sesiones de hemodiálisis desarrolla una observación continua del paciente, previniendo muchas complicaciones existentes. En este sentido, se destaca la actuación de los enfermeros al establecer de forma precoz los diagnósticos de enfermería (DE) y la implementación de las intervenciones adecuadas ${ }^{(2)}$.

Para garantizar el perfecto restablecimiento del paciente es imprescindible la implementación de las fases del proceso de la Sistematización de la Asistencia en Enfermería (SAE) por la enfermera, destacando que uno de los aspectos cruciales de su actuación en la unidad de hemodiálisis es la prestación de cuidados sistematizados basados en un referencial teórico ${ }^{(5)}$.

No obstante, el proceso de desarrollo de la SAE, que también constituye un instrumento de acercamiento entre enfermero y paciente, choca a menudo con la estructura de las unidades que prestan el servicio, en virtud del pequeño número de profesionales responsables por un gran número de pacientes por sesión, provocando la no realización del proceso de enfermería ${ }^{(5)}$.

Aunque muchos enfermeros hayan encontrado dificultades en algunas instituciones para la implementación de la SAE, se denota a través de los resultados de estudios científicos que la implantación efectiva, en general ,basada en una taxonomía de la North American Nursing Diagnosis Association Internacional (NANDA I), corresponde a las necesidades de los pacientes renales crónicos fundamentalmente, garantizando la calidad en la asistencia prestada ${ }^{(5,6)}$.

La enfermería consciente debe actuar en la prevención y control de complicaciones, además de estar atenta a los aspectos biopsicosociales vividos por el sujeto foco de su cuidado, desarrollando su actuación de manera más eficiente con la implementación de la SAE en su práctica diaria.

De esta forma, este estudio tuvo como objetivo determinar los diagnósticos de enfermería basados en la nomenclatura de la North American Nursing Diagnosis Association - Internacional (NANDA I) 2009-2011 ${ }^{(7)}$, más frecuentes en pacientes sometidos al tratamiento de hemodiálisis.

\section{MÉTODO}

Se realizó un estudio cuantitativo, de tipo descriptivo exploratorio en una clínica de hemodiálisis, en un municipio del interior del estado de São Paulo.

La técnica del muestreo fue del tipo no probabilístico por conveniencia, en el que se incluyeron todos los pacientes mayores de 18 años sometidos al tratamiento de hemodiálisis por medio de la fístula arteriovenosa (FAV) que firmaron el Término de Consentimiento Libre e Informado, tras recibir informaciones sobre los objetivos del estudio, garantía de anonimato y la libertad de desistir en cualquier momento de la realización del mismo. 
Se consideraron como criterios de exclusión, los pacientes en hemodiálisis por catéter doble lumen, que presentaban problemas psiquiátricos 0 , aun, aquellos con dificultades de comunicación.

Para la recogida de datos, se realizaron visitas al sector de hemodiálisis de la clínica en día y horario determinados por la institución, reservadas previamente con los enfermeros responsables por el sector, en el periodo de febrero a abril de 2010.

Para la etapa inicial de la investigación, se empleó el instrumento de recogida de datos, que tuvo como eje orientativo para su elaboración la Teoría del Autocuidado de Orem, propuesto por Araújo (2003) en su tesina de maestría ${ }^{(8)}$. Este instrumento consiste en un historial de enfermería detallado, que contiene aspectos relativos a la salud del paciente, limitaciones impuestas por la enfermedad y examen físico. Tras la recolecta del historial de enfermería, los datos provenientes se organizaron, analizaron e identificaron los diagnósticos según la clasificación de la NANDA I 2009-2011 ${ }^{(7)}$.

El estudio fue aprobado por el Comité de Ética en Investigación de la Universidad del Vale de Paraíba (CEP-UNIVAP) bajo número de protocolo H236/CEP/2009 del 24 de febrero de 2010.

\section{RESULTADOS}

Se incluyeron 50 individuos, 27 (54\%) eran del sexo masculino y $23(46 \%)$ del sexo femenino, con edades de entre 20 y 70 años, siendo que 17 (34\%) del muestreo estaban entre los 41 y los 50 años (Tabla 1). Entre los hombres la edad media fue de 55,8 años y para las mujeres, 50,5 años.

En relación al estado civil, 39 (78\%) viven con sus respectivos compañeros (Tabla 1).

La enfermedad crónica de mayor relevancia fue la Hipertensión Arterial Sistémica (HAS) observada en 38 (76\%) de los pacientes. La mayoría de los individuos investigados (94\%) no eran fumadores o consumidores frecuentes de alcohol (Tabla 1).

El periodo de realizacón de la diálisis por los pacientes varió de entre un mes y 13 años, con 18 (36\%) de los sujetos en tratamiento entre 6 y 10 años (Tabla 1). El tiempo medio de tratamiento fue de 4 años y 10 meses.

Tabla 1 - Distribución de las variables socio-demográficas de los 50 pacientes investigados. SP, 2010.

\begin{tabular}{lcc}
\hline Variables & $\mathrm{n}$ & $\%$ \\
\hline Sexo & & \\
Masculino & 27 & 54 \\
Femenino & 23 & 46 \\
Edad (años) & & \\
$20-30$ & 5 & 10 \\
$31-40$ & 1 & 2
\end{tabular}




\begin{tabular}{lcc}
\hline $41-50$ & 17 & 34 \\
$51-60$ & 13 & 26 \\
$61-70$ & 8 & 16 \\
70 o + & 6 & 12 \\
Estado civil & & \\
Soltero (a) & 3 & 6 \\
Casado (a) & 39 & 78 \\
Separado (a) & 4 & 8 \\
Viudo (a) & 4 & 8 \\
Enfermedades pre-existentes & & \\
HAS & 38 & 76 \\
HAS+ Diabetes Mellitus & 12 & 24 \\
Antecedentes personales & & \\
Tabaquismo & 3 & 6 \\
Etilismo & 0 & 0 \\
Tiempo de diálisis & & \\
1 mes - 1 año & 12 & 24 \\
2 - 5 años & 13 & 26 \\
$6-10$ años & 18 & 36 \\
11 años o + & 7 & 14 \\
\hline
\end{tabular}

Considerando los casos estudiados, se identificaron 24 diagnósticos de enfermería (DE) diferentes, según la clasificación NANDA I 2009-2011, siendo seis de los mismos observados en $100 \%$ del muestreo estudiado, que son: riesgo de desequilibrio electrolítico, eliminación urinaria deficiente, movilidad física limitada, riesgo de perfusión renal ineficaz, riesgo de infección e integridad de la piel afectada (Tabla 2).

Se observó que de los 24 diagnósticos de enfermería, 17 (70,8\%) se clasifican como reales: volumen de líquidos excesivo, eliminación urinaria afectada, constipación, patrón de sueño alterado, desplazamiento deficiente, movilidad física alterada, fatiga, intolerancia a la actividad, percepción sensorial disminuida (visual), memoria insuficiente, patrones de sexualidad ineficaces, comportamiento de salud propenso al riesgo, falta de adhesión, dentición afectada, integridad de la piel afectada, mucosa oral afectada y dolor agudo; y siete $(29,2 \%)$ eran diagnósticos de riesgo: riesgo de glicemia inestable, riesgo de desequilibrio electrolítico, riesgo de constipación, riesgo de perfusión renal ineficaz, riesgo de baja autoestima situacional, riesgo de infección y riesgo de caída (Tabla 2).

Tabla 2 - Distribución de los diagnósticos de enfermería encontrados en 50 pacientes investigados. SP, 2010.

\begin{tabular}{lcc}
\hline Diagnóstico de Enfermería & $\mathrm{N}$ & $\%$ \\
\hline Riesgo de desequilibrio electrolítico & 50 & 100 \\
Eliminación urinaria afectada & 50 & 100 \\
Movilidad física disminuida & 50 & 100 \\
Riesgo de perfusión renal ineficaz & 50 & 100 \\
Riesgo de infección & 50 & 100 \\
Integridad de la piel afectada & 50 & 100
\end{tabular}


Volumen de líquidos excesivo

Mucosa oral afectada

Riesgo de constipación

Percepción sensorial perturbada (visual)

Dentición afectada

Fatiga

Padrones de sexualidad ineficaces

Intolerancia a actividad

Padrón de sueño alterado

Comportamiento de salud propenso a riesgo

Memoria afectada

Dolor agudo

Riesgo de baja autoestima situacional

Constipación

Riesgo de glicemia inestable

Falta de adhesión

Desplazamiento afectado

Riesgo de caídas

Respecto a los dominios predominantes entre los diagnósticos realizados, se destaca el número 4, relativo a la actividad y reposo con seis caos (25\%); seguido por el dominio 11, relativo a seguridad y protección del paciente, que representa cinco $(20,8 \%)$ de los casos. Los dominios nutrición y eliminación/cambio, presentaron tres diagnósticos (12,5\%) cada uno (Tabla 3).

Tabla 3 - Distribución de diagnósticos de enfermería según sus dominios. São José dos Campos, 2010.

\begin{tabular}{lcc}
\hline Dominios & $\mathrm{N}$ & $\%$ \\
\hline 2 - nutrición & 3 & 12,5 \\
3 - eliminación/cambio & 3 & 12,5 \\
4 - actividad/reposo & 6 & 25 \\
5 - percepción/cognición & 2 & 8,3 \\
- auto-percepción & 1 & 4,2 \\
8 - sexualidad & 1 & 4,2 \\
9 - enfrentamiento/tolerancia al estrés & 1 & 4,2 \\
10 - principios de la vida & 1 & 4,2 \\
11 - seguridad/protección & 5 & 20,8 \\
12 - comodidad & 1 & 4,2 \\
\hline Total & 24 & 100 \\
\hline
\end{tabular}

Se optó por determinar los principales factores relacionados y características definidoras o factores de riesgo de los seis DE presentados por todos los sujetos investigados, habiéndose presentado de forma separada en diagnósticos reales (Cuadro 4) y de riesgo (Cuadro 5) para una mejor comprensión. 
Cuadro 4 - Presentación de los Diagnósticos de Enfermería reales presentes en todo el muestreo estudiado, principales características definidoras y principales factores relacionados. SP, 2010.

\begin{tabular}{cll}
$\begin{array}{c}\text { Diagnósticos de } \\
\text { Enfermería }\end{array}$ & \multicolumn{1}{c}{$\begin{array}{c}\text { Características } \\
\text { definidoras }\end{array}$} & \multicolumn{1}{c}{$\begin{array}{c}\text { Factores } \\
\text { relacionados }\end{array}$} \\
\hline $\begin{array}{c}\text { Eliminación urinaria } \\
\text { afectada }\end{array}$ & - Disuria; & - Múltiples causas. \\
& - Retención urinaria. & \\
\hline Movilidad física afectada & - Amplitud limitada de & - Fuerza muscular \\
& movimientos; & reducida; \\
& - Movimientos lentos; & - Intolerancia a actividad; \\
& - Capacidad limitada para & - Restricciones \\
& desempeñar habilidades & prescritas de \\
& motoras básicas. & movimientos. \\
\hline & & - Factores mecánicos; \\
Integridad de la piel & - Rompimiento de la & - cambio en el estado \\
afectadrico; & superficie de piel. & - Nutrición \\
& & desequilibrada; \\
& & - Cambio de la turgencia.
\end{tabular}

Cuadro 5 - Presentación de los Diagnósticos de Enfermería presentes en todo el muestreo estudiado y sus principales factores de riesgo. SP, 2010.

\begin{tabular}{ll}
\hline Diagnósticos de Enfermería & Factores de riesgo \\
\hline Riesgo de desequilibrio electrolítico & - Disfunción renal; \\
& - Desequilibrio hídrico. \\
\hline & - Enfermedad renal; \\
& - Hipertensión; \\
Riesgo de perfusión renal ineficaz & - Diabetes Mellitus; \\
& - Edad avanzada; \\
& - Efectos secundarios relacionados al \\
& tratamiento \\
\hline Riesgo de infección & - Procedimientos invasivos; \\
& - Enfermedad crónica. \\
\hline
\end{tabular}

\section{DISCUSIÓN}

La hemodiálisis es un tratamiento de sustitución del filtraje sanguíneo, paliativo, pues no recupera integralmente la salud del paciente. El enfermero por medio del levantamiento de diagnósticos de enfermería, crea la sistematización individualizada del trabajo, ofreciendo una mejor calidad del vida al paciente ${ }^{(9)}$.

Los resultados del presente estudio no coinciden con la literatura científica reciente, que apunta un predominio de hombres de entre 41 y 50 años sometidos a hemodiálisis, y que se abstienen de la ingestión de bebidas alcohólicas, puesto que el alcohol puede contribuir a la elevación de la tensión arterial y con un tiempo medio de realización del tratamiento de diálisis de 4 años y 10 meses, similar al tiempo mencionado en estudio anterior, de 3 años y 2 meses $^{(9)}$. 
Los DE aquí identificados tampoco coinciden con la visión de los reconocidos en otras investigaciones entre pacientes renales crónicos ${ }^{(4,9,10)}$. Como por ejemplo en el estudio que analizó los DE de pacientes sometidos a trasplante renal en tratamiento de ambulatorio, de entre los 38 diagnósticos de enfermería identificados, seis equivalen a los mismos diagnósticos identificados en este estudio, de entre los 10 que se encontraban por encima del percentil 75, que son: riesgo de infección, percepción sensorial afectada (visual), padrón de sueño afectado, fatiga, dolor agudo y padrones de sexualidad ineficaces ${ }^{(10)}$.

Así, en la presente investigación, seis DE se referían al dominio actividad y reposo, destacándose en este grupo el diagnóstico de movilidad física afectada presente en toda la población; en este punto conviene destacar las complicaciones provenientes de la diabetes y otras limitaciones de la enfermedad.

Frecuentemente la FAV trae alteraciones en el día a día de los pacientes renales crónicos en virtud de la limitación de movimientos posibles y en muchas situaciones, se requiere la realización de nuevas operaciones para obtención de otros $_{\text {accesos }}^{(11)}$.

Tres DE encontradas se referían al dominio nutrición y entre ellos, el de riesgo de desequilibrio de volumen de líquidos estuvo presente en todo el muestreo investigado y volumen de líquidos excesivo en $84 \%$ de los sujetos. Estudio reciente señaló la hiperhidratación como un problema frecuente en las unidades de hemodiálisis provocando el incremento de la tensión arterial y consecuentes complicaciones durante el procedimiento ${ }^{(12)}$.

De esta forma se observa el empleo frecuente de antihipertensivos en muchos centros de tratamiento, mientras otros alían la restricción dietética de sal, en la tentativa de minimizar esta realidad. Estudio reciente acompañó durante un año dos grupos, uno $(n=190)$ sometido a restricción de sal y otro $(n=204)$ con el empleo de agentes hipertensivos. Se concluyó que los pacientes sometidos a restricción dietética de sal utilizaron sustancialmente menos agentes hipertensivos y tuvieron menos incremento de peso inter-dialítico ${ }^{(13)}$.

Por lo tanto, la enfermería debe ser importante aliada del paciente en la tentativa de proponer y reforzar medidas que les permitan controlar mejor su nutrición, en relación a la sal, líquidos y nutrientes.

En este sentido, reforzando los DE encontrados sobre riesgo de constipación y constipación, una investigación procuró evaluar el incremento de peso interdialíctico y su relación con mala nutrición. Los autores no encontraron correlaciones significativas entre el incremento de peso inter-dialíctico e indicadores de mala nutrición en la población joven, aunque en la población mayor de 65 años, el incremento del peso inter-dialíctico estuvo asociado a la ingestión insuficiente de alimento, desorden gastrointestinal, incapacidad funcional, acompañado por pérdidas musculares y de grasa en ancianos ${ }^{(14)}$.

Reforzando los aspectos relativos a la ingestión de alimentos, destacamos los DE de mucosa oral y dentición afectadas. El estudio evaluó la salud oral de pacientes renales crónicos sometidos a tratamientos conservadores (a través de medicación y dieta) y hemodiálisis presentaron menor prevalencia del caries, aunque tengan 
una placa sobre las encías, mayor cantidad de dientes con pérdida de inserción y pérdidas dentarias, comparados con la población sana (controles) ${ }^{15}$.

Ante estas constataciones y conscientes de la carencia de informaciones y acceso de la población a servicios de odontología, el enfermero deberá establecer vínculos con los servicios públicos de sanidad odontológica, atendiendo a este aspecto de la población, que puedo comprometer su calidad de vida y encaminarlos hacia un seguimiento adecuado, reflejándose en mejoría de los aspectos nutritivos de los pacientes en hemodiálisis.

En la secuencia, otros dos diagnósticos que enfocan todo el muestreo investigado fueron el de eliminación urinaria afectada, perteneciente al dominio eliminación y cambio, y el de riesgo de perfusión renal ineficaz, del dominio actividad y reposo.

Muchas veces la producción de orina cesa completamente en pacientes sometidos a diálisis, aunque muchos pacientes presenten presencia de orina residual, definida como la producción de por lo menos $250 \mathrm{ml}$ de orina diarios. Investigadores intentaron determinar la asociación de presencia de orina con calidad de vida, mortalidad e inflamación por medio de un estudio de corte. De entre los 734 pacientes investigados, el $84 \%$ presentó orina residual, considerando que la función residual de riñón estuvo asociado a una mejor sobrevida y calidad de vida, menor porcentaje de inflamación y utilización de eritropoyetina. Sugiriendo que esta información debe ser constantemente monitoreada en pacientes sometidos a hemodiálisis ${ }^{(16)}$.

Otro aspecto destacable es el control de la ingestión hídrica, una de las complicaciones más frecuentes identificadas, asociada al incremento de peso inter-dialítico, y que debe observarse por el equipo ${ }^{(4)}$.

Una vez más la enfermería asume un papel fundamental en el adecuado mantenimiento del tratamiento, que se refleja significativamente en la calidad de vida de los pacientes, pues como se señala en este y en otra investigación sobre el asunto, muchos de los diagnósticos observados en esta categoría de pacientes están asociados a la dificultad de seguimiento del tratamiento exigido por la enfermedad $^{(17)}$.

Dentro del dominio seguridad y protección se destaca el DE de riesgo de infección e integridad de la piel afectada.

La FAV es el principal acceso vascular para el paciente en tratamiento de hemodiálisis y su mantenimiento depende de los cuidados prestados por el equipo de enfermería y del paciente ${ }^{(4)}$.

Las múltiples punciones incrementaron el riesgo de infección, requiriendo que la enfermería esté atenta con las características del acceso ideal, que debe poseer adecuado flujo sanguíneo para la realización de la diálisis, garantizando una larga vida útil y como consecuencia, bajas complicaciones ${ }^{(4)}$. Además, se observa habitualmente FAV que no funcionan, cicatrices presentes en los accesos actuales y cicatrices de fístulas perdidas ${ }^{(18)}$.

EI DE percepción sensorial disminuida (visual) afectó al 64\% del muestreo investigado en la presente investigación, la reducción de la acuidad visual está 
relacionada muchas veces con la caída de la calidad de vida, afectando a las actividades diarias de los pacientes y caídas accidentales. Estudio anterior observó que el 95,6\% de los pacientes sometidos a hemodiálisis con más de 65 años presentaron acuidad visual en niveles menores de los esperados para su edad $^{(19)}$.

De esta forma, se imagina que la actuación preventiva de la enfermería respecto a las alteraciones visuales prevendrá otros DE detectados, como deambulación reducida y riesgo de caídas, que afectaron respectivamente al 14\% del muestreo de la presente investigación.

El tratamiento de los pacientes renales crónicos aun hoy impone diversas complicaciones, convirtiendo de esta forma el establecimiento de los diagnósticos e intervenciones de enfermería en imprescindibles para la obtención de una mejor calidad de vida ${ }^{(9)}$.

El enfermero se destaca como coordinador del equipo y debe atender a las necesidades individuales del paciente, garantizando un trabajo multidisciplinario y una mejor adecuación al tratamiento de hemodiálisis, puesto que aunque el enfrentamiento de la enfermedad renal sea solitario, desarrollan a lo largo del tratamiento una convivencia intensa con el equipo de enfermería ${ }^{(20)}$.

\section{CONCLUSIÓN}

Los principales diagnósticos evidenciados en la presente investigación fueron: eliminación urinaria afectada, integridad de la piel afectada, riesgo de infección, riesgo de perfusión renal ineficaz, movilidad física afectada y riesgo de desequilibrio electrolítico.

Se denota así que los pacientes renales crónicos presentan diversos diagnósticos de enfermería en común, exigiendo del profesional de enfermería una planificación para atender las necesidades de cuidado de los pacientes.

Para ello, el enfermero debe emplear la SAE para planear e implementar sus cuidados de forma sistematizada, evaluando continuamente este proceso.

El presente estudio permitió una reflexión respecto a las características comunes a los pacientes sometidos a tratamiento de hemodiálisis, que generaron diagnósticos propuestos en esta investigación, lo que ayudará al profesional de enfermería implicado en la atención a los pacientes renales crónicos, proporcionando herramientas para la planificación de la asistencia.

\section{REFERENCIAS}

1. Higa K, Kost MT, Soares DM, Morais MC, Polins BRG. Qualidade de vida de pacientes portadores de insuficiência renal crônica em tratamento de hemodiálise. Acta Paul Enferm. 2008;21(n. especial):203-6.

2. Nascimento CD, Marques IR. Intervenções de enfermagem nas complicações mais freqüentes durante a sessão de hemodiálise: Revisão de literatura. Rev Bras Enferm. 2005 nov-dez;58(6):719-22. 
3. Barbosa GS, Valadares GV. Experimentando atitudes e sentimentos: o cotidiano hemodiálitico como base para o cuidar em enfermagem. Esc Ana Nery Rev Enferm. 2009;13(1):17-23.

4. Souza EF, De Martino MMF, Lopes MHBM. Diagnóstico de enfermagem em pacientes com tratamento hemodialítico utilizando o modelo teórico de Imogene King. Rev Esc Enferm USP. 2007;41(4):629-35.

5. Oliveira SM, Ribeiro RCHM, Ribeiro DF, Lima LCEQ, Pinto MH, Poletti NAA. Elaboração de um instrumento da assistência de enfermagem na unidade de hemodiálise. Acta Paul Enferm. 2008;21(n. especial):169-73.

6. Bisca MM, Marques IR. Perfil de diagnósticos de enfermagem antes de iniciar 0 tratamento hemodialítico. Rev Bras Enferm. 2010 mai-jun;63(3):435-9.

7. North American Nursing Diagnosis Association. Diagnósticos de Enfermagem da NANDA: definições e classificações 2009-2011. Porto Alegre: Artmed; 2010.

8. Araújo MOPH. O auto cuidado em idosos independentes residentes em instituições de longa permanência [dissertação]. Campinas (SP): Faculdade de Ciências Médicas, Universidade Estadual de Campinas; 2003. $280 f$.

9. Lata AGB, Albuquerque JG, Carvalho, LASBP, Lira ALBC. Diagnósticos de enfermagem em adultos em tratamento de hemodiálise. Acta Paul Enferm. 2008;21 (n. especial):160-3.

10. Lira ALBC, Albuquerque JG, Lopes MVO. Perfil dos diagnósticos de enfermagem presentes em pacientes transplantados renais. Rev Enferm UERJ. 2007 jan-mar;15(1):13-9.

11. Corrêa JA, Pires AC, Kafejian O, Miranda Jr. F, Galego S J, Yamazaki Y R, et al. Fístula arteriovenosa safeno-femoral superficial como acesso à hemodiálisedescrição de técnica operatória e experiência clínica inicial. J Vasc Bras [periódico na internet]. 2005; [citado 2011 jan 13]; 4(4):341-8. Disponível em: http://www.scielo.br/scielo.php?script=sci_arttext\&pid=S1677-

54492005000400008

12. Machek P, Jirka T, Moissl U, Chamney $P$, Wabel P. Guided optimization of fluid status in haemodialysis patients. Nephrol Dial Transplant. 2010 Feb;25(2):538-44.

13. Kayikcioglu M, Tumuklu M, Ozkahya M, Ozdogan O, Asci G, Duman S, et al. The benefit of salt restriction in the treatment of end-stage renal disease by haemodialysis. Nephrol Dial Transplant. 2009;24:956-962.

14. Yang SC, Chiang CK, Hsu SP, Hung KY. Relationship between interdialytic weight gain and nutritional markers in younger and older hemodialysis patients. $\mathrm{J}$ Ren Nutr. 2008 Mar;18(2):210-22.

15. Marinho JSS, Carmona IT, Loureiro A, Posse J L, Caballero LG, Dios PD. Oral health status in patients with moderate-severe and terminal renal failure. Med oral patol oral cirbucal [periódico na internet]. 2007 ago[citado 2011 jan 13];12(4):[aprox. 8 telas] Disponível em: http://scielo.isciii.es/scielo.php?script=sci_arttext\&pid=S169869462007000400008 \&lng=es\&nrm=iso\&tlng=en\&tlng=en

16. Shafi T, Jaar BG, Plantinga LC, Fink NE, Sadler JH, Parekh RS, et al. Association of residual urine output with mortality, quality of life, and inflammation in incident hemodialysis patients: the Choices for Healthy Outcomes in Caring for End-Stage Renal Disease (CHOICE) Study. Am J Kidney Dis. 2010 Aug;56(2):348-58.

17.Costa AGS, Santos RMB, Vitor AF, Araujo TL. Diagnósticos de enfermagem de pacientes em tratamento de hemodiálise em hospital-escola. Rev Enferm UFPE on line[periódico na internet]. 2010 jul-set[acesso em 2012 fev 29];4(3):1477-83. 
http://www.ufpe.br/revistaenfermagem/index.php/revista/article/view/1047/pdf_126 18.Nascimento VPC, Abud ACF, Inagaki ADM, Daltro AST, Viana LC. Avaliação da técnica de curativo em cliente com acesso venoso para hemodiálise. Rev Enferm UERJ. 2009 abr-jun;17(2):215-9.

19. Chiu E; Markowitz SN; Cook WL; Jassal SV Visual impairment in elderly patients receiving long-term hemodialysis. Am J Kidney Dis. 2008 Dec;52(6):1131-8.

20. Dyniewicz AM, Zanella E, Kobus LSG. Narrativa de um cliente com insuficiência renal crônica: a história oral como estratégia de pesquisa. Rev Eletr Enf[periódico na internet]. 2004[acesso em 2011 fev 29];6(2):199-212. Disponível em: http://www.fen.ufg.br/revista/revista6_2/pdf/Orig7_narrativa.pdf 